\title{
Intraocular Foreign Body in the Posterior Chamber
}

\author{
Hana Park ${ }^{\mathrm{a}}$, Jae Hyung Lee ${ }^{\mathrm{a}}$, Ho Ra ${ }^{\mathrm{a}}$, b
}

\begin{abstract}
Intraocular foreign bodies may not cause any symptoms and there are some case reports about intralenticular foreign bodies and foreign bodies in anterior angle or posterior segment that were retained in the orbit for many years. We report a case of intraocular foreign body found in the posterior chamber.
\end{abstract}

Keywords: Intraocular foreign body; Ocular trauma; Posterior chamber

\section{Introduction}

Intraocular foreign body can be a great threat to vision and the orbit itself. Occasionally the site of penetration may not be obvious, and the clinician should presume the mechanism of trauma and the presence of foreign body in the orbit. We report a case of metallic intraocular foreign body found in the posterior chamber.

\section{Case Report}

A 48-year-old male presented to our ophthalmology department with irritation sensation of his right eye. He described that while hammering an iron-containing metal-on-metal 10 days ago, he felt a piece of metal hit his right eye. He visited the local clinic the next day, and there were no pathologic findings on the initial examination. He began to experience foreign body sensation, discharge and injection of the right eye, and decided to visit our clinic as the symptoms got worse.

\footnotetext{
Manuscript accepted for publication July 07, 2014

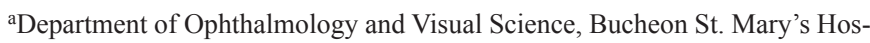
pital, College of Medicine, The Catholic University of Korea, Seoul, Korea ${ }^{b}$ Corresponding Author: Ho Ra, Bucheon St. Mary's Hospital, College of Medicine, The Catholic University of Korea, 2 Sosa Dong, Wonmi Gu, Bucheon, Kyunggi Do 420-717, Korea. Email:raho@catholic.ac.kr
}

doi: http://dx.doi.org/10.14740/jmc1855w
On initial examination, the patient's visual acuity was $20 / 25$ in right eye and 20/20 in left eye. In his right eye, there was a healed $1 \mathrm{~mm}$ corneal laceration nasal to the visual axis, and the Seidal test was negative. The conjunctiva was moderately injected with chemosis and the anterior chamber was deep with rare inflammatory cells. The iris appeared round and regular, and no foreign body was found on gonioscopic evaluation of the right eye. The anterior lens capsule of the right eye showed anterior surface opacity but no sign of penetration was present. The patient's fundus examination showed no definite abnormality. We persuaded the patient to take a plain orbit Xray just in case, which later showed a tiny radioopaque lesion in the right orbit. A computed tomography (CT) scan of orbits was performed to rule out the possibility of intraorbital foreign body and a $1.5 \mathrm{~mm}$ metallic foreign body was embedded in the inferior area of posterior chamber (Fig. 1).

We planned a surgical removal under topical anesthesia. A clear corneal incision was made at the 12 o'clock position. Viscoelastic material (healon) was injected into the anterior chamber, and three iris retractors were applied at the 6 o'clock position. When the foreign body was not revealed with iris retractors, we made the patient to look downward. A metallic foreign body was identified in the space between the zonule and the iris. Healon was instilled around the foreign body, shifting it to the center of the anterior chamber (Fig. 2). The foreign body was carefully removed with forceps. Postoperatively, there were no specific findings except trace cell reactions in the anterior chamber. Inflammatory reaction of the conjunctiva was subsided with eyedrops (moxifloxacin, prednisolone). The patients's final visual acuity was $20 / 25$ postoperatively.

\section{Discussion}

There are some points to be noted in our case. Slit lamp examinatioin is used to detect any pathologic findings of the eye by ophthalmologists. In our case, possibility of foreign body in the orbit can be overlooked because none was found in slit-lamp examination. There was a corneal scar but we may presume that the foreign body fell out by itself, and the lens opacity may be confused as cataract. There are blind spots that cannot be detected by slit-lamp examination, one of which demonstrated by our case is behind the iris. Although the pupils of the patient were fully dilated, we could not observe the edge of the space 


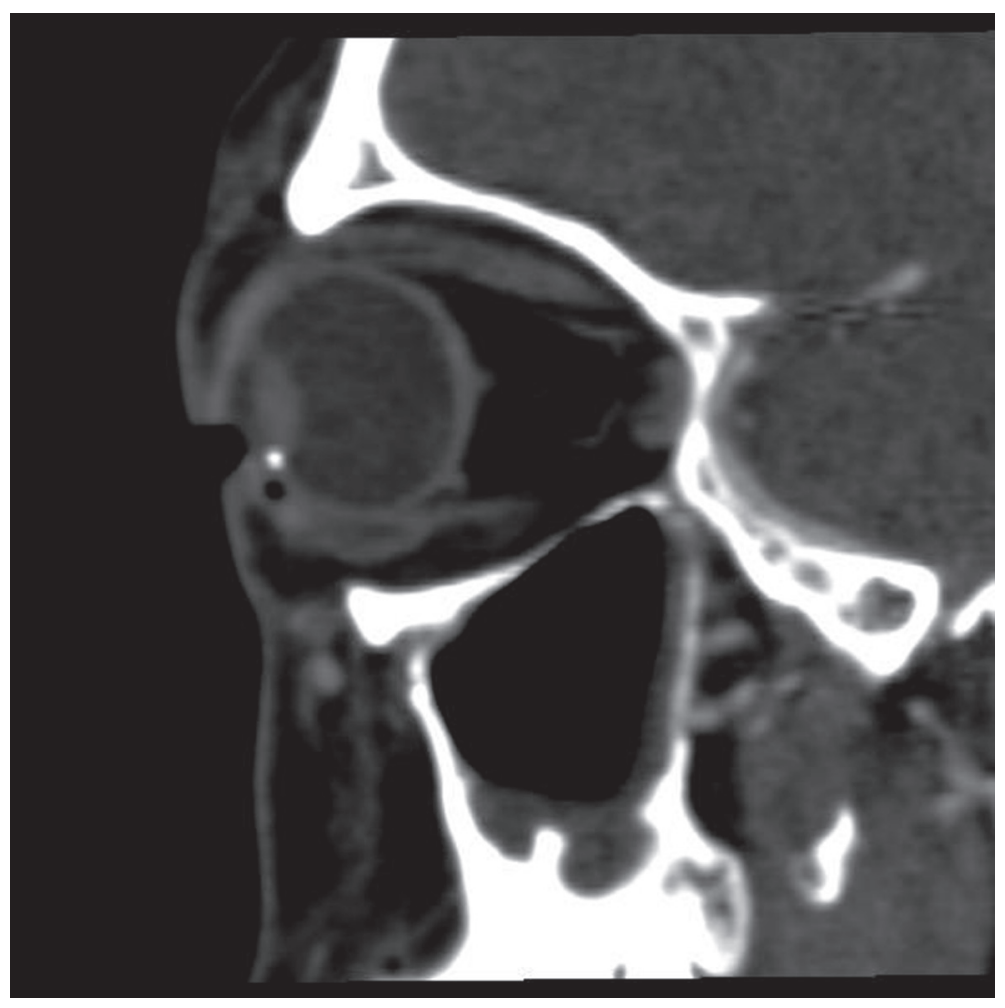

Figure 1. Metallic foreign body found on CT scan.

between the iris and the ciliary body by slit-lamp examination.

Few cases of intraocular foreign bodies presenting in the anterior chamber angle have been reported. In this case, the foreign body appeared to have entered through the cornea, hit the anterior lens capsule and then "fell" into the inferior angle [1]. In our case, the location seems to be the posterior chamber, and such case has never been reported.

Foreign body in posterior chamber may not be detected in initial examination even though early detection and removal is essential in the management of intraocular foreign body. Infectious endophthalmitis is a severe vision-threatening complication of an intraocular foreign body. Most study series cite such endophthalmitis incidence between $4.7 \%$ and $13.3 \%$, depending on the study [2]. The risk of endophthalmitis is decreased if the intraocular foreign body is removed within the first $24 \mathrm{~h}$ after the injury [3].

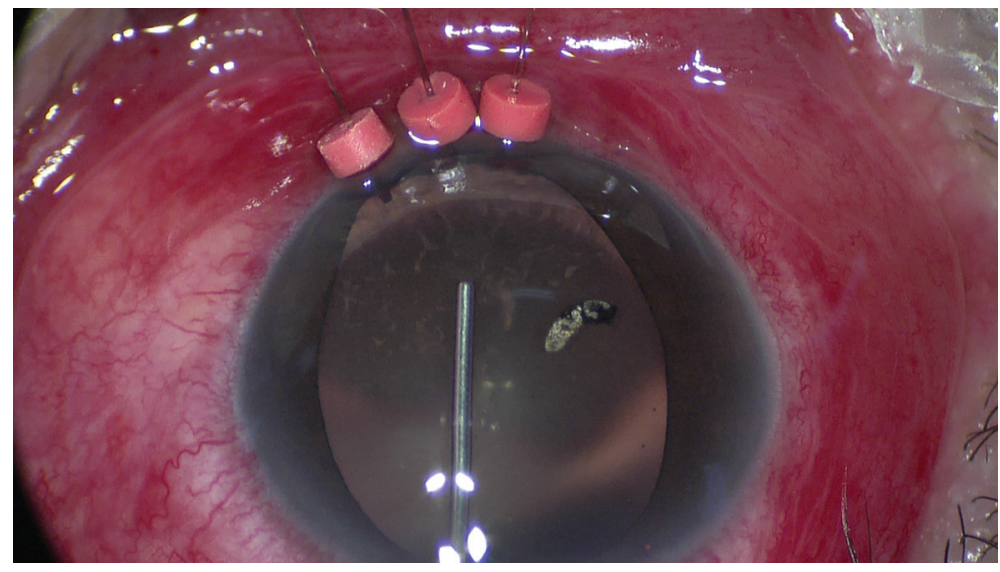

Figure 2. Foreign body removal by instilling healon. 
Our case demonstrates that ophthalmologist should be highly suspicious of the presence of an intraocular foreign body when examining a patient who experienced ocular trauma.

Imaging study such as plain X-ray or CT should be performed when intraocular foreign body was not detected in ocular examination in patients who have a history of trauma and signs of penetration.

\section{Disclosure}

The authors have no proprietary interest related to this article.

\section{References}

1. Davidson RS, Sivalingam A. A metallic foreign body presenting in the anterior chamber angle. CLAO J. 2002;28(1):9-11.

2. Thompson JT, Parver LM, Enger CL, Mieler WF, Liggett $\mathrm{PE}$. Infectious endophthalmitis after penetrating injuries with retained intraocular foreign bodies. National Eye Trauma System. Ophthalmology. 1993;100(10):14681474.

3. Jonas JB, Budde WM. Early versus late removal of retained intraocular foreign bodies. Retina. 1999;19(3):193197. 